\title{
Effect of Cognitive Behavioral Intervention on Stress Response of Nurses with PTSD in Operating Room
}

\author{
Yi Huang, Yajuan Ke*, Hongying Zhu, Yunsuo Gao, Duozhi Wu, Qiu Cai, Juncheng Guo \\ Hainan General Hospital, Haikou, China \\ Email: *g2002m@163.com
}

How to cite this paper: Huang, Y., Ke, Y.J., Zhu, H.Y., Gao, Y.S., Wu, D.Z., Cai, Q. and Guo, J.C. (2021) Effect of Cognitive Behavioral Intervention on Stress Response of Nurses with PTSD in Operating Room. World Journal of Neuroscience, 11, 8-16. https://doi.org/10.4236/wjns.2021.111002

Received: January 18, 2021

Accepted: January 30, 2021

Published: February 2, 2021

Copyright $\odot 2021$ by author(s) and Scientific Research Publishing Inc. This work is licensed under the Creative Commons Attribution International License (CC BY 4.0).

http://creativecommons.org/licenses/by/4.0/

(c) (i) Open Access

\begin{abstract}
Objective: To investigate the effect of cognitive behavioral intervention on nurses with PTSD by using cognitive behavioral intervention. Methods: The subjects were selected by randomized control method, and the PTSD nurses were divided into control group $(\mathrm{n}=30)$ and intervention group $(\mathrm{n}=30)$. The intervention group used cognitive behavioral intervention; the control group did not use cognitive behavioral intervention. Nurses with PTSD in the two groups completed the basic situation survey, medical coping questionnaire (MCMQ) and anxiety self-rating form (SAS) psychological scale one month later. Results: There was no significant difference in MCMQ and SAS scores between the two groups before intervention $(\mathrm{P}>0.05)$. After intervention, the scores of MCMQ and SAS in the intervention group were compared with the scores of MOCQ and SAS in the control group. The scores of the control group after intervention were significantly higher than those of the intervention group, with statistical significance $(\mathrm{P}<0.05)$. There were statistically significant differences in MCMQ and SAS in the intervention group before and after intervention $(\mathrm{P}<0.05)$. There was no statistical significance in the control group before and after intervention $(\mathrm{P}>0.05)$. It shows that cognitive behavioral intervention therapy has the effect and value of improving the psychological status of nurses with PTSD. Conclusion: Cognitive behavioral intervention therapy combined with psychology for nurses with PTSD can effectively relieve their stress level, relieve their mood, and improve their positive coping ability and work efficiency.
\end{abstract}

\section{Keywords}

Stress Response, Cognitive Behavioral Therapy, Operating Room Nurse, Mental Health 


\section{Introduction}

Operating room is an important place for the rescue of dangerous, urgent and serious patients, and also a place for various surgical treatments. It occupies a very important position in the hospital. The development of various surgical operations cannot be separated from the close cooperation of operating room nurses. With the change of medical model and the rapid development of science and technology, the work content of operating room nurses covers a wider range, which has higher requirements for the quality of operating room nursing, but also brings great pressure to the operating room nurses' psychology. Operating room nurses, as a special group in the nursing team, undertake the operation cooperation of the whole surgery. The operation process is long and continuous, resulting in irregular life of operating room nurses, work overload, and the disorder of the human biological clock. In addition to the basic theories and knowledge of nursing, operating room nurses also need to have a certain grasp and understanding of the operation related knowledge of various departments and different systems of human body, such as instruments and anatomical knowledge, so as to be able to cooperate with different operations. In terms of emergency concept and ability, operating room nurses also have higher requirements than other departments [1] [2]. In 1975, the Association of Operation Room Nurses (AORN) pointed out that the work of operating Room Nurses is not only the nursing during surgery [3], but also the perioperative nursing in three stages, including a series of processes such as preoperative visit, intraoperative nursing cooperation, postoperative anesthesia recovery and so on. With the continuous development of medical science and technology, the work content of operating room nurses is constantly increasing, the scope of work is constantly expanding, the degree of work is constantly improving, and the depth and breadth of knowledge that need to be mastered are constantly expanding. With the continuous development of medical science and technology, the work content of operating room nurses is constantly increasing, the scope of work is constantly expanding, the degree of work is constantly improving, and the depth and breadth of knowledge that need to be mastered are constantly expanding. Operating room work is busy, fast pace and heavy responsibility, the risk of medical errors will increase. With the continuous improvement of medical level, the level of hospital medical service is more and more demanding, and the operation is more and more elaborate. New and complex medical equipment continues to enter the hospital operating room, which requires nursing staff to be familiar with the operating procedures of medical equipment, and more importantly, it is necessary for nurses in the operating room to update their professional knowledge and improve their operating skills. Therefore, operating room nurses are always in a state of concentrated energy. Without strong psychological quality, they are prone to psychological anxiety, leading to serious job burnout and so on. Most of the study of the operating room nurses also showed that the workload is that most of the nurses were investigated, mentioned, followed 
by the worry about accidents. The nursing work cannot get the understanding and sympathy of patients, the medical workload is large, the working hours are irregular, doctors and nurses sometimes have differences because of the work reasons, and there will be misunderstandings between colleagues; nursing managers have insufficient understanding and support for nursing work, great competition for professional promotion, poor working environment, fear of infection, etc., these factors make the operating room nurses often feel great psychological and professional pressure [4] [5] [6], and most of the operating room nurses are still predominantly female, male is complementary. While working, women have to bear family burdens such as caring for their husband, raising their children and taking care of their elders. The irregularity of operating room work makes them have no time to take care of their families, which may cause negative psychological reactions. Whether there are differences in psychological reactions between male and female medical workers needs to be analyzed in future studies. Relevant domestic research shows that the quality of life of operating room nurses in China is lower than that of the general population, especially the scores in many fields such as physiology, psychology and environment are low. The greater the work pressure, the worse the psychological status, and the incidence of depression and anxiety symptoms is significantly higher than that of the general staff and other nursing professionals in China [7] [8] [9]. A large number of studies have confirmed that operating room nurses have a higher incidence of mental diseases compared with nurses in routine clinical departments. At present, the research on the psychological stress state of operating room nurses is still insufficient, and the occurrence of PTSD is worth exploring. This paper intends to use cognitive behavioral intervention to intervene the stress response of nurses with PTSD in the operating room, and evaluate the effect of the intervention.

\section{Research Objects and Methods}

\subsection{Subjects}

In this study, post-traumatic stress disorder (PTSD) tests were conducted on operating room nurses in a number of "Grade A" hospitals in Hainan Province from 2019 to 2020. By using symptoms of PTSD-civilian version (PCL-C) and clinical diagnosis, nurses diagnosed with PTSD were randomly divided into control group and intervention group with 30 cases in each group. All enrolled nurses signed the informed consent, and the study was approved by the ethics committee of the hospital.

\subsubsection{Inclusion Criteria}

1) Nurses working in hospital operating rooms; 2) Older than 18 - 25 years old;

3) No history of other serious diseases and mental illness; 4) Basic reading and writing ability, able to complete the questionnaire independently, and willing to participate in this research. 


\subsubsection{Exclusion Criteria}

1) Nurses who are not engaged in hospital operating rooms; 2) Nurses older than 26 years or younger than 18 years; 3) suffer from mental illness; Patients with underlying diseases and cancers; 4 ) Those who cannot complete the scale test and refuse to participate in the research work.

\subsection{Research Tools}

\subsubsection{Self-Rating Anxiety Scale (SAS) [10]}

A self-rated psychological scale used to reflect and analyze the subjective feelings of patients with anxiety symptoms and to measure the severity of their anxiety status as well as changes in patients during treatment. A rough score is obtained by adding up the scores of each of the 20 items. Multiply the rough fraction by 1.25 to take the whole part. I get a standard score. The result was a standard score, with the higher the score, the more severe the symptoms. Generally speaking, the total score of anxiety is less than 50 points for normal; People with mild anxiety range from 51 to 60 , moderate anxiety range from 61 to 70 , and severe anxiety range from 71 to over. The number of negative items indicates how many items the subjects did not respond to, and the number of positive items indicates how many items the subjects responded to.

Note: in the 20 items of SAS, the scores of items 5, 9, 13, 17 and 19 must be calculated in reverse. The reverse calculation means that the scores are scored in the opposite frequency.

SAS is simple to use and can be guided by self-evaluators without special training. Moreover, it is quite convenient to analyze and can effectively reflect the related symptoms and their severity and changes of anxiety state, especially suitable for screening anxiety state in general hospitals. SAS scores are not affected by age, gender, financial status and other factors. It is now widely used.

\subsubsection{Medical Coping Modes Questionnaire (MCMQ) [11]}

A scale used to reflect a patient's coping style and ability in the event of a major illness. The scale currently used has been partially modified by domestic psychologists on the basis of the original scale. The scale contains 20 items, which are mainly divided into three dimensions: "facing", "avoiding" and "yielding". MMCMQ uses a four-level scale to assess the severity of symptoms in each item. There are 8 items marked with ${ }^{*}$ in the whole scale for reverse evaluation, and the scoring standard adopts reverse score from back to front. The corresponding items of the three dimensions were respectively accumulated and added to calculate the mean value of the corresponding quantities.

\subsection{Research Methods}

\subsubsection{Sampling}

Subjects meeting the inclusion criteria were randomly divided into intervention group and control group by convenient sampling method. The control group did not receive cognitive behavioral intervention therapy intervention, the interven- 
tion group received cognitive behavioral intervention therapy for psychological crisis intervention and psychological guidance.

\subsubsection{Intervention Methods}

In intervention group cognitive behavior intervention methods [12], mainly includes: 1) cognitive reconstruction: using rational emotional therapy, behavior therapy on PTSD nurses with cognitive factors, use the rational thinking method to replace PTSD nurse irrational thinking method, reduce PTSD nurses caused by irrational thinking all kinds of negative emotions and bad ways. 2) Behavioral therapy: for overly anxious PTSD nurses, progressive muscle relaxation therapy should be added in the intervention process to relieve the tension of PTSD nurses. Progressive muscle relaxation therapy mainly uses PTSD nurses to consciously tension and relaxes the muscle groups in various parts of the body, so that PTSD nurses can eliminate tension from the whole body and slowly achieve a purpose of relaxation, so as to adjust and relieve the stress response of PTSD nurses and promote the harmony of body and mind. After the identification of the intervention objects, psychological intervention was given to the subjects once 35 - 45 min every day before the operation, and the intervention time was 0.5 months. In the intervention process, investigators try to be sincere, avoid using too many professional terms, encourage PTSD nurses from time to time, and create a pleasant and relaxed atmosphere for conversation, so that PTSD nurses can understand and actively cooperate with this study.

\subsubsection{Statistical Methods}

SPSS21.O statistical software was used to input, sort out and analyze the data. Statistical methods included descriptive statistical analysis, independent sample, test, analysis of variance, correlation analysis and multiple linear regression analysis.

\section{Results}

\subsection{Comparison of SAS Scores of PTSD Nurses in the Two Groups before and after Intervention}

Paired sample $t$ test was used to statistically analyze the SAS scores of PTSD nurses in the two groups before the intervention, and there was no statistical significance in the SAS scores between the intervention group and the control group before the intervention $(P>0.05)$. After intervention, the SAS scores of the intervention group and the control group were significantly different $(\mathrm{P}<$ 0.05). Before and after the intervention, the SAS scores of the intervention group were significantly different $(\mathrm{P}<0.05)$. There was no significant difference in SAS scores in the control group before and after intervention $(P>0.05)$. See Table 1 for details.

\subsection{Comparison of MCMQ Subscales Scores of PTSD Nurses in the Two Groups before and after Intervention}

Pair sample $t$ test was used to analyze the scores of three different coping styles 
Table 1. Comparison of anxiety scores of nurses with PTSD between two groups before and after intervention $(\bar{x} \pm s)$.

\begin{tabular}{ccccc}
\hline Project & $\begin{array}{c}\text { The intervention } \\
\text { group } \\
(\mathbf{n}=\mathbf{5 0})\end{array}$ & $\begin{array}{c}\text { The control } \\
\text { group } \\
(\mathbf{n}=50)\end{array}$ & $\mathbf{t}$ & $\mathbf{P}$ \\
\hline $\begin{array}{c}\text { Anxiety scores before } \\
\text { intervention } \\
\text { Anxiety scores after } \\
\text { intervention }\end{array}$ & $45.78 \pm 5.67$ & $46.02 \pm 5.67$ & 0.210 & 0.417 \\
$\mathrm{t}$ & $38.29 \pm 5.75$ & $45.76 \pm 7.85$ & & \\
$\mathrm{P}$ & 6.559 & 0.189 & 5.428 & 0.000 \\
\hline
\end{tabular}

Note: $P<0.05$ was considered statistically significant.

of PTSD nurses in the two groups before intervention, and there was no statistical significance in the scores of "facing", "avoiding" and "yield" between the intervention group and the control group before intervention $(P>0.05)$. After intervention, the scores of "facing" and "avoiding" coping styles were significantly different between the intervention group and the control group $(\mathrm{P}<0.05)$. Before and after intervention, there were statistically significant differences in the MCMQ scores of "facing" and "avoiding" two coping styles in the intervention group $(\mathrm{P}<0.05)$. There was no significant difference in the MCMQ scores of the control group before and after intervention $(P>0.05)$. See Table 2 for details.

\section{Discussion}

\subsection{Influence of Cognitive Behavioral Intervention on Anxiety of Nurses with PTSD}

From the experimental results of this study show, before the intervention scores of anxiety in the intervention group and control group not significant, after combining the psychology of cognitive behavioral intervention, intervention group and control group for anxiety score significantly lower anxiety scores before intervention, while the control group anxiety scores also fell, but the intervention group anxiety score fell more obvious. Before the intervention, the anxiety difference between the two groups was not statistically significant, but after the intervention, the anxiety difference between the two groups was statistically significant. The difference of anxiety in the intervention group before and after the intervention was statistically significant, while that in the control group was not statistically significant. The results of the study indicate that cognitive behavioral intervention has a good effect on improving the anxiety of nurses with PTSD and is an effective intervention measure, which is basically consistent with the research results of domestic and foreign scholars using cognitive behavioral intervention therapy on emotional intervention of nurses with PTSD [13] [14] [15]. 
Table 2. Comparison of scores of each subscale of PTSD nurses' medical coping styles between the two groups before and after intervention $(\bar{x} \pm s)$.

\begin{tabular}{|c|c|c|c|c|c|}
\hline \multicolumn{2}{|c|}{ Medical coping style } & $\begin{array}{l}\text { The intervention group } \\
\qquad(\mathrm{n}=50)\end{array}$ & $\begin{array}{l}\text { The control group } \\
(\mathrm{n}=50)\end{array}$ & $\mathbf{t}$ & $\mathbf{P}$ \\
\hline \multirow{4}{*}{$\begin{array}{l}\text { In the } \\
\text { face of }\end{array}$} & Before the intervention & $17.92 \pm 3.77$ & $18.67 \pm 7.90$ & 0.606 & 0.273 \\
\hline & After the intervention & $23.24 \pm 5.63$ & $17.74 \pm 5.06$ & 5.138 & 0.000 \\
\hline & $\mathrm{t}$ & 5.552 & 0.701 & & \\
\hline & $\mathrm{P}$ & 0.000 & 0.242 & & \\
\hline \multirow{4}{*}{ avoid } & Before the intervention & $15.64 \pm 2.69$ & $16.03 \pm 2.31$ & 0.778 & 0.219 \\
\hline & After the intervention & $13.53 \pm 1.78$ & $16.31 \pm 1.93$ & 7.487 & 0.000 \\
\hline & $\mathrm{t}$ & 4.625 & 0.658 & & \\
\hline & $\mathrm{P}$ & 0.000 & 0.256 & & \\
\hline \multirow{4}{*}{ yield } & Before the intervention & $8.07 \pm 6.35$ & $8.96 \pm 5.51$ & 0.749 & 0.228 \\
\hline & After the intervention & $7.73 \pm 6.01$ & $8.03 \pm 6.41$ & 0.241 & 0.405 \\
\hline & $\mathrm{t}$ & 0.275 & 0.778 & & \\
\hline & $\mathrm{P}$ & 0.392 & 0.219 & & \\
\hline
\end{tabular}

Note: $P<0.05$ was considered statistically significant.

\subsection{Influence of Cognitive Behavioral Intervention on Medical Coping Styles of Nurses with PTSD}

The results of this study showed that the three medical coping styles of the PTSD nurse intervention group and the control group had the same dimensions of "facing", "avoiding" and "yielding", which were balanced and comparable between the groups. Before intervention, there were no statistically significant differences in the "face", "avoid" and "yield" of the medical coping styles between the PTSD nurses in the intervention group and the control group [16]. After the cognitive behavioral intervention, the "face" coping style of PTSD nurses in the intervention group was gradually enhanced with the advance of the intervention therapy, while the "avoidance" and "yield" coping styles were gradually decreased with the advance of the intervention therapy. This result shows that, with the progress of intervention treatment, cognitive behavioral intervention therapy can effectively improve the medical coping style of nurses with PTSD to psychological stress, reduce the "avoidance" coping style, reduce the degree of "avoidance", and improve the "face" coping style [17] [18] [19]. This result is similar to the research results of domestic and foreign scholars using cognitive behavioral therapy on the intervention of medical coping style of nurses with PTSD. The reason may be that PTSD nurses lack of knowledge about the disease, fear and high expectations about the examination, treatment effect and prognosis of the disease. In addition to the pain of the disease itself, nurses with PTSD also have to endure psychological pain, so they are more prone to produce fragile emotions. With the increase of work pressure, the greater the impact of their bad stress mode on negative psychology, leading to PTSD nurses that are more 
inclined to adopt an "avoidance" coping mode. After some psychological intervention, PTSD nurses in the intervention group could take the initiative to recognize their own bad coping styles, take the initiative to adjust, and adopt a more positive "face" coping style to receive treatment [20] [21] [22] [23].

Through the MCMQ and SAS scores of nurses with PTSD, this study found that active cognitive behavioral intervention can effectively reduce the stress response of nurses with PTSD and improve the medical coping style of nurses with PTSD. Reasonable intervention therapy has good application value for clinical practice and is suitable to be promoted to clinical departments. Operating room nurses are a special nursing team whose work is at the core of the surgical system. However, current research on the condition of the nurse career remaining blank spots, especially in the operating room nurses remains to be further perfect, therefore, the purpose of this paper is to discuss the operating room nurses psychological capital, work the pressure source and the current situation of career status and before both influence on career status, but due to the limitation of time and place, this study only in a hospital operating room nurse intervention experiment, the sample range is too small, limited sample size. Moreover, due to the influence of subjective factors, the intervention measures in this study only adopted cognitive reconstruction and behavioral therapy of progressive muscle relaxation training, while other intervention methods were not involved. Therefore, further research and discussion are still needed. Moreover, the human resources combined with the clinical knowledge and psychological knowledge of hospital medical staff are limited and have not yet reached the conditions for comprehensive promotion. Therefore, this cognitive behavioral intervention measure can only be gradually promoted in clinical application.

\section{Funding}

The present study was supported by the Key Technology Project in Hainan, China (No. ZDYF2019159).

\section{Conflicts of Interest}

The authors declare no conflicts of interest regarding the publication of this paper.

\section{References}

[1] Liu, Y. and Zhang, J.Y. (2005) Investigation and Analysis of Work Burnout of Operating Room Nurses. China Nursing Management, 5, 44-46.

[2] Zhu, D. and Zhou, L. (2008) Nursing in Operating Room. People's Medical Publishing House, Beijing.

[3] Chang, P.L., Chou, Y.C. and Cheng, F.C. (2007) Career Needs, Career Development Programmes, Organizational Commitment and Turnover Intention of Nurses in Taiwan. Journal of Nursing Management, 15, 801-810. https://doi.org/10.1111/j.1365-2934.2007.00772.x

[4] Hu, W.B. (2009) Research on the Relationship between Job Stressors, Type A Per- 
sonality and Job Burnout in Nurses. Tianjin Normal University, Tianjin.

[5] Cai, G.F. (2009) Investigation and Analysis of Working Stress Sources of Operating Room Nurses. Journal of Nursing Science, 24, 30-31.

[6] Mc Abee, R. (1991) Occupational Stress and Burnout in the Nursing Profession. AAIHN Journal, 39, 568. https://doi.org/10.1177/216507999103901204

[7] Pan, L.Y., He, R.B., Liu, C.H., et al. (2015) Typical Correlation Analysis of Operating Room Nurses' Quality of Life and Its Influencing Factors. Medical Equipment, 30, 118-121.

[8] Ling, S.Q. and Guo, X.H. (2006) Investigation and Analysis on Mental Health Status of Nurses in Operating Room. Contemporary Nurses, 2, 92-94.

[9] Mgallery, Li, J., Bai, H.X., et al. (2012) Effect of Group Psychological Training on Nursing Staff in General Hospital. Journal of Modern Integrated Chinese and Western Medicine, 20, 472-474.

[10] Zhang, M.Y. (1993) Handbook of Psychiatric Rating Scale. Hunan Science and Technology Press, Changsha, 38-41.

[11] Wang, X.D., Wang, X.L., et al. (1999) Manual of Mental Health Rating Scale. Chinese Journal of Mental Health, 31-35, 124-131.

[12] Qu, Y.M. and Yang, D.M. (2015) Pay Attention to Stress Psychological Training and Maintain the Physical and Mental Health Development of Middle School Students. Reading, Writing and Computing: Teachers' Edition, 40, 1-4. (In Chinese)

[13] Li, D.Z., Li, X.L. and Yang, L.H. (2004) Investigation on Stressors and Coping Styles of Operating Room Nurses. Nursing Journal of Science, 19, 62-64.

[14] Zhao, H.Y. (2010) Analysis on the Physical and Mental Health Problems of Operating Room Nurses. Chinese Practical Nursing Miscellaneous Chi, 26, 66-67.

[15] Lin, Q.Q. (2010) Psychological Stress and Countermeasures of Operating Room Nurses. Chinese Journal of Traditional Chinese Medicine Emergency, 19, 723-724.

[16] Xiao, L.L., Zhuang, S.L. and Yu, X.Y. (2011) Investigation and Analysis of Psychological Stress and Psychological Needs of Nurses in Operating Room. Hainan Medical Science, 22, 150-151.

[17] Peng, W.H. and Qiu, J.F. (2014) Improving Social Support System to Promote Psychological Harmony of Operating Room Nurses. Chinese Journal of Disability Medicine, 22, 254-255.

[18] Zhao, H.J., Liu, X.F. and Hou, J.L. (2013) Analysis of Symptoms and Related Factors of Post-Traumatic Stress Disorder in Operating Room Nurses. Modern Nursing, 24, 166-168.

[19] Wu, F.H. (2009) Analysis and Discussion on Psychological Stress of Operating Room Nurses. Management and Education, No. 3, 123-125.

[20] Zhu, M. (2004) Analysis of the Current Research Status of Nurses' Mental Health in China from 133 Literatures. Chinese Journal of Nursing, 39, 215-216.

[21] Lin, L. and He, G.P. (2006) Epidemiological Characteristics and Risk Factors of Post-Traumatic Stress Disorder in Emergency Department Nurses. Modern Nursing, 12, 1592-1594.

[22] Zeng, J. (2015) Correlation Analysis of Working Stress and Coping Style of Operating Room Nurses and Their Physical and Mental Health. Medical Information, 28, 263.

[23] Chang, Q., Li, S. and Zhou, Y. (2008) A Study on the Relationship between Occupational Life Events and Job Burnout of Operating Room Nurses. Nursing Research, 22, 1257-1239. 